\title{
Spontaneous echo-contrast in LV non-ischemic dilated cardiomyopathy in sinus rhythm. What therapy to prevent cardio-embolic events?
}

\author{
Federico Cacciapuoti* \\ - Department of Cardiovascular diseases at University of Naples, Italy
}

\begin{abstract}
Dilatative Cardiomyopathy (DC) with low Ejection Fraction \% (EF \%> 35\%), Spontaneous EchoContrast (DEC) in the Left Ventricle (LV) seen at echocardiography and in sinus rhythm predisposes to the intraventricular thrombus formation and subsequently embolization, resulting in stroke. Current guidelines recommend oral vitamin $\mathrm{K}$ antagonists (VKAs) to prevent brain's cardio-embolic events deriving from SEC present in LV, as previously evidenced in several trials (HELAS, WASH, WACH, WARCEF). This treatment to prevent embolic stroke is effective both for ischemic and non-ischemic DC with SEC in LVN and in sinus rhythm. But, in the future, could be timely to treat with new oral anticoagulants (NOACs) non-ischemic DC alone, whereas this prophylactic treatment adopted in ischemic DC (already treated with antiplatelet drugs) could raise the risk of minor or major bleedings.

Keywords: Dilatative Cardiomyopathy in sinus rhythm and low ejection fraction; spontaneous echocontrast; echocardiography.
\end{abstract}

\section{Introduction}

Dilated Cardiomyopathy (DC) is a progressive disease of cardiac chambers, with prevalence of systolic dysfunction of left ventricle (LV) that becomes enlarged and cannot pump blood efficaciously. It is the third most common cause of heart failure and the most frequent cause of heart transplantation. The term is employed to indicate an idiopathic or familial DC (primitive DC). Other known causes are: chronic hypertension, valvular diseases, toxic exposure (cobalt), chronic alcoholism, autoimmune diseases, and the use of certain drugs or pregnancy (secondary DC) [1]. But, DC with low ejection fraction\% (EF\%) secondary to coronary artery disease, even though is the most frequent secondary DC [2], must be excluded from our debate.

It is known that DC with low EF\% of LV favors the aggregates of red blood cells and fibrinogen. Instead, the platelets do not participate to this reduction because aren't echogenic [3-5]. The interaction between erythrocytes and plasma proteins causes a slowing down of hematic fluidity, responsible of increased echogenicity.
That appears (at echocardiography) such as spiral of smoke, also named as spontaneous echo-contrast (SEC). Usually, SEC is often present in non-valvular atrial fibrillation (NVAF) and can cause a risk of thromboembolic cerebral events antagonized by novel oral anticoagulant drugs (NOACs) [6]. But, SEC can also be evident in LV-DC patient in sinus rhythm [7].

In the present review, we debated about the causes of cerebral embolism originating by SEC of non-ischemic LV of DC in sinus rhythm.

\section{Spontaneous echo-contrast (SEC)}

The phenomenon is evident both at trans-thoracic and trans-esophageal echocardiography (fig.1), such as swirling smoke-like image associated with reduced blood

Corresponding Author: Federico Cacciapuoti, Department of Cardiovascular diseases at University of Naples, Italy E-mail: fulviocacciapuoti@gmail.com Received date: April 08, 2018; Accepted date: April 27, 2018; Published date: April 30, 2018.

DOI: https://doi.org/10.31546/JCCCVT.1001 
swirling flow or stasis [8, 9]. The imaging has been considered as pre-thrombotic state predisposing to thrombo-embolic stroke [10]. Evidence of hypercoagulable state in patients with heart failure with low EF\% is supported by elevated levels of von Willebrand factor, increased platelet's activation and heightened markers of thrombin production [11]. Specifically, fibrinopeptide A and thrombin-antithrombin complex positively correlated with LV end-diastolic volume and negatively with fractional shortening of LV [12]. Conditions favoring thrombus-formation in the left ventricle are: advancing age, surgery, especially of knee and hip, cancers, immobilization, obesity, severe infections, pregnancy or oral use of contraceptives. These act by increasing pro-coagulant factors without concomitant increase in natural anticoagulant proteins [13].

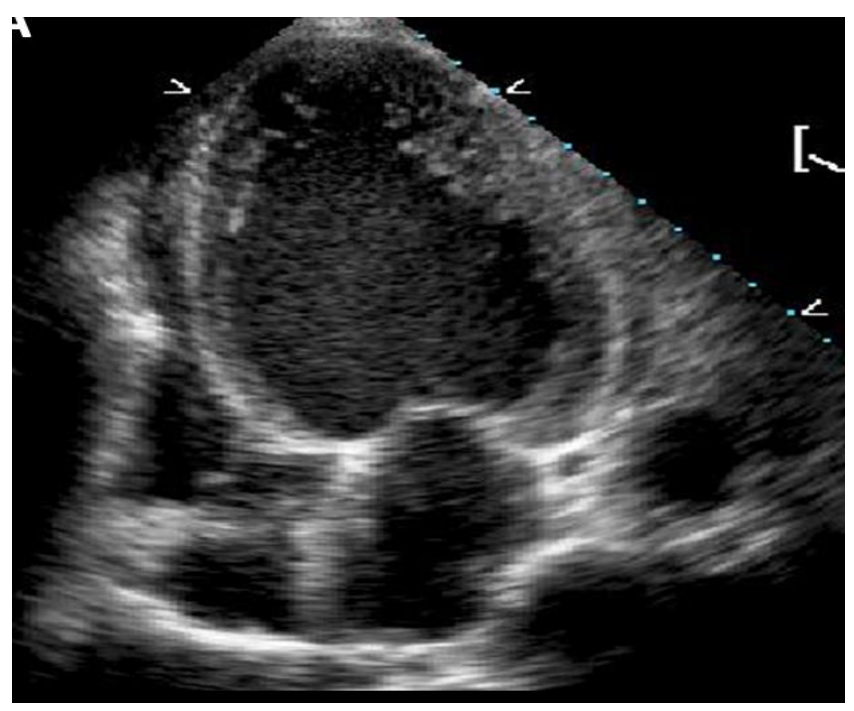

Fig.1-2DE-Evidence of spontaneous echo-contrast in the left ventricle in dilated cardiomyopathy.

\section{Previous studies}

In DC with low EF\% and SEC in LV seen at echocardiography, an increased percentage of thromboembolic events (especially stroke) were found [14].

In a study performed with trans-thoracic echocardiography, SEC in LV was present in $11 \%$ of the patients with DC. Concordantly in another trial, thromboembolic accidents were found with higher prevalence in DC-patients in sinus rhythm having SEC than in those without [15]. The underlying cardiac conditions were: ischemic heart disease in $68 \%$ of cases; non-ischemic cardiomyopathy in $19.3 \%$ and valvular heart disease in $12.5 \%$.
In this study it is evident that the presence of SEC in LV increases the occurrence of cardio-embolic events of $10 \%$ compared to $1.8-2.4 \%$ obtained in patients with left ventricular dysfunction without SEC. Similar results were also reported in another previous study [16]. Finally, stroke and other cardio-embolic events occurred in $16.7 \%$ of patients in sinus rhythm, DC with low EF\% and SEC comparatively with $4.6 \%$ of patients having DC, low EF\%, but without SEC [17].

\section{Antiplatelets vs. Anticoagulants}

Considerable controversies there are regarding the role of anticoagulants compared with antiplatelet drugs to reduce the incidence of thrombo-embolic accidents in patients with DC in sinus rhythm, low Ejection Fraction and SEC seen at echocardiography. Previously, the Heart Failure Long-term Antithrombotic Study (HELAS) performed in patients with DC of ischemic or idiopathic aetiology and treated with warfarin, aspirin or placebo, noted that no overall significant difference between anticoagulants and antiplatelets there are as regard the incidence of embolic events in the groups evaluated, even if warfarin was lightly more effective [18].

In the WASH (Warfarin/Aspirin Study in Heart failure) study, no significant difference in death, stroke, or myocardial infarction was found among 279 patients enrolled. However, the highest rate of re-hospitalization was among those receiving aspirin [19].

Concordantly, the Warfarin and Antiplatelet Therapy in Chronic Heart failure (WATCH) trial has recently demonstrated that warfarin was not superior to aspirin and clopidogrel also was not superior to aspirin in to prevent cardio-embolic events. However, the highest rate of re-hospitalization was among those receiving aspirin, whereas warfarin lowered the percentage of embolic stroke, but at the cost of an increased hemorrhage rate [20]. Finally, the Warfarin versus Aspirin in Reduced Cardiac Ejection fraction (WARCEF) study, more recently, confirmed that there was little differences in stroke and death from any cause between warfarin and aspirin. But, in this study also the greater reduction of cardio-embolic accidents obtained with warfarin was counterbalanced by an increased risk of major hemorrhage [21].

The reported studies were performed both in ischemic and non-ischemic DC. The results obtained underline that antiplatelets are essential to treat both coronary ischemia (responsible for ischemic DC), and 
possible embolic events. But, the prevention of these (reported in non-ischemic DC) happens less frequently with antiplatelet drugs in comparison to warfarin, even though at expense of increased hemorrhage rate. Concordantly, referring to the recent therapeutic suggestions, in non-ischemic DC in sinus rhythm with SEC in LV, oral anticoagulant vitamin $\mathrm{K}$ antagonists (VKAs) is preferable to antiplatelet drugs in to prevent possible cardio-embolic events.

\section{Novel Oral Anti-Coagulants (NOACs)}

But, taking into account the overall superiority of Novel Oral Anti-Coagulants (NOACs) over VKAs [22], the risk of possible cardio-embolic accidents could be further reduced with undeniable advantages rising from the employment of NOACs instead of VKAs. In fact, NOACs are an actractive option for DC in sinus rhythm, with SEC seen in LV [23].

Compared with VKAs, NOACs have more advantages, such as: the VKA dose is determined on an individual basis (not fixed), whereas NOACs are administered in fixed doses, except when a patient has a functional disorder of the liver or kidney. Other advantages of NOACs over VKAs are their efficacy in preventing stroke and thromboembolisms in patients with atrial fibrillation. Conversely, their efficacy in DC with SEC and sinus rhythm wasn't experienced too. In addition, NOACs over VKAs have lower incidence of major bleeding, minor drug and food interactions, rapid onset and offset action, short half-time and lack of the need for laboratory monitoring. However, some disadvantages of NOACs must be also mentioned, such as their costs and the absence of specific antidotes [24]. DC with low EF\% and SEC in LV in sinus rhythm predisposes to intraventricular thrombi. That subsequently evolves in embolization resulting in stroke. This condition requires NOACs prevention, analogously to non-valvular atrial fibrillation (NVAF). In fact, the echocardiographic SEC in LV is indicative of the thrombi evolving in brain's emboli, even in sinus rhythm [25].

\section{Conclusive remarks}

At moment, DC patients with heart failure, low EF\% $(<35 \%)$ and in sinus rhythm are treated with warfarin [26]. The treatment recommendations are based on the results of previous randomized, controlled trials, as HELAS, WASH, WASCH, and WARCEF. But, a wide, randomized, controlled trial could be performed in the future, to verify if VAKs therapy can be advantageously replaced by NOACs, to prevent the brain's emboli originating from LV of DC in sinus rhythm, when echocardiography shows SEC. But, this treatment should be performed in non-ischemic DC with echocardiographic evidence of SEC in LV and in sinus rhythm alone.

The antiplatelet therapy to be performed for coronary disease not suggests the contemporary NOACs giving in patients with ischemic DC, low $\mathrm{EF} \%$ and sinus rhythm to prevent the embolic stroke. In fact, even if the concomitant use of antiplatelet agents and NOACs is increasing in clinical practice, several data suggest that patients already treated with aspirin and/or clopidogrel, who additionally receive NOACs show a 3 -fold increase in the risk of haemorrhage without further reduction of adverse coronary events. Thus, an individualized approach is requested in a patient contemporarily receiving antiplatelets and NOACs, balancing the potential benefit against the increase risk of haemorrhage [27-29].

\section{Statement}

Informed Consent: Informed consent was not necessary, because none patient was evaluated in the study.

Ethical Approval: All procedures performed were in accordance with the ethical standards of the institutional and/or national research committee and with the 1964 Helsinki declaration and its later amendments or comparable ethical standards.

\section{Conflicts of Interest: None}

\section{References:}

1. Maron BJ, Towbin JA. et al. Contemporary definitions and classification of the cardiomyopathies: an American Heart Association Scientific Statement from the Council on Clinical Cardiology Heart Failure and Transplantation Committee; Quality of Care and Outcomes Research and Functional Genomics and Translational Biology Interdisciplinary Working Group and Council on Circulation 2006; 113, 1807-1016.

2. Boffa GM, Tarantini G, Abbasciano A. et al. Ischemic cardiomyopathy: lack of clinical applicability of the WHO/SFC classification of cardiomyopathies. Ital. Heart J. 2001; 2, 778-781. 
3. Zhdanova SG, Petrikov SS. et al. Dilated cardiomyopathy as a cause of ischemic stroke. $\mathrm{Zh}$. Nevrol. Psikhiatr. Im. SS. Korsakova 2016; 116, 44-47.

4. Sharma ND, McCullogh PA, Weaver WD Left ventricular thrombus and subsequent thromboembolism in patients with severe systolic dysfunction. Chest 2000; 117, 314-320.

5. Rastegar R, Harnick DJ. et al. Spontaneous echocontrast videodensity is flow-related and is dependent on the relative concentrations of fibrinogen and red blood cells. J. Am. Coll. Cardiol. 2003; 41, 603-.610.

6. Inoue $\mathrm{H}$. Thromboembolism in patients with non valvular atrial fibrillation: comparison between Asian and Western countries. Int. J. Cardiol. 2013; 61, 1-7.

7. Merino A, Hauptman P, Badimon L. et al. Echocardiographic "smoke" is produced by an interaction of erythrocytes and plasma proteins modulated by shear forces. J. Am Coll. Cardiol. 1992; 20, 1661-1668.

8. Armstrong WF, Ryan T. Feigenbaum's Echocardiography, $7^{\text {th }}$ edition. Philadelphia Lippincott Williams \& Wilkins. 2010.

9. Kupczynka K, Kaspzak JD. et al. Prognostic significance of spontaneous echocardiographic contrast detected by trans-thoracic and trans-esophageal echocardiography in the era of harmonic imaging. Arch. Med. Sci. 2013; 9, 808-814.

10. Esmon CT: Basic mechanisms and pathogenesis of venous thrombosis- Blood Rev. 2009; 23, 225-229.

11. Gibbs CR, Blann AD. et al. Abnormalities of hemoreological endothelial and platelet functions in patients with chronic heart failure in sinus rhythm: effects of angiotensin-converting enzyme inhibitor and beta blocker therapy. Circulation 2001; 103, 1746-1751.

12. Ikeda U, Yamamoto K, Shimada K.: Biochemical markers of coagulation activation in mitral stenosis, atrial fibrillation and cardiomyopathy. Clin. Cardiol. 1997; 20, 7-10.

13. Mahajan N. Ganguly J, Simeon M, Bhattacharya P, Shankar L, Mahavan R, Chaurvedi S, Ramappa P, Alfonso L. Predictors of stroke in patients with severe systolic dysfunction in sinus rhythm: Role of echocardiography. Int. Journ. Cardiol. 2010; 145, 8789.
14. Chiong LL, Tumabiene KD, Vincente MA, Abelardo NS. Prevalence of cardio-embolic event among patients with spontaneous echo contrast on trans-thoracic echocardiography (SMOCC-Heart study). Phil. Journ. Int. Med. 2014; 52, 1-5.

15. Dans AL, Morales DD, Velandria F, Abola TB, Roxas Jr. A, Punzalan ER, Allyn R, Tachev E, Amarillo L, Villaruz MV. National Nutrition and Health Survey (NNHS) Atherosclerosis-Related diseases and risk factors. Phil. Journ. Cardiol. 2005; 33, 103-115.

16. Dries DL, Rosenberg TD, Waclawiw MA, Domanski MJ. Ejection Fraction and risk of thromboembolic events in patients with systolic dysfunction and sinus rhythm: evidence for gender differences in the studies of left ventricular dysfunction trials. Journ. Amer. Coll.Cardiol. 1997; 29, 1074-1080.

17. Freudenberg RS, Halperin JL.: Should we use anticoagulation for patients with chronic heart failure? Nat. Clin. Prat. Cardiovasc. Med. 2006; 3, 580-581.

18. Cokkinos PK, Haralabopoulos GC, Kostis JR, Toutozas PK. Efficacy antithrombotics therapy in chronic heart failure: the HELAS study. Eur, J. Heart Fail.2006; 8, 428-432.

19. Cleland JGF, Finday I, Jafri S, Sutton G, Falk R, Bulpitt C, Prentice C, Ford I, Trainer A, Poole-Wilson PA. The Warfarin/Aspirin Study in Heart failure (WASH): a randomized trial comparing antithrombotic strategies for patients with heart failure. Am. Heart J. 2004; 148, 157-164.

20. Massie BM, Collins JF, Ammon SE, et al. Randomized trial of warfarin, aspirin, and clopidogrel in patients with chronic heart failure: the Warfarin and Antiplatelet Therapy in Chronic Heart failure (WATCH) trial. Circulation 2009; 119, 1616-1624.

21. Homma S, Thomson JL, Pullicino PM, Levin B, Freudenberger RS, Teerlink JR, Ammon SE, Graham S, Sacco RL, Mann D. et al. Warfarin and aspirin in patients with heart failure in sinus rhythm. N. Engl. J. Med. 2012; 366, 1859-1869.

22. Elkeboom JW, Weitz JL. New anticoagulants. Circulation 2010; 121, 1523-1532.

23. Arbit B, Hsu JK. Non-vitamin K antagonist oral anticoagulant use in patients with atrial fibrillation and associated intracranial hemorrhage: a focused review. Clin. Cardiol. 2015; 38, 681-691. 
24. Mekay H, Mekay A, Duci JB, Miftari EI. New oral anticoagulants: their advantages compared with vitamin $\mathrm{K}$ antagonists in the prevention and treatment of patients with thromboembolic events. Ther. Clin. Risk Management 2015; 11, 967-977.

25. Sivri N, Yetkin E, Tekin GO, Yalta K, Waltenberger J. Anticoagulation in patients with left ventricular systolic dysfunction and sinus rhythm: when? Clin. Appl. Thromb. Hemost. 2014; 20, 729-734.

26. Yaney CM, Jesup M, Bozkurt B, Butler J, Casey DE, Drazner MH, Fonarw GC, Geraci SA, Hozwich T, et al. ACCF/AHA guideline for the management of heart failure: A report of the American College of Cardiology
Foundation/American Heart Association task force on practice guideline. Circulation 2013; 128, e240-e327.

27. Eikelboom JW, Hirh J. Combined antiplatelet and anticoagulant therapy: clinical benefits and risks. J. Thromb. Hemost. 2007; 5, 255-263.

28. Oldgren J, Wallentin L, Alexander JH, Jones S, Jonelid B, Steg G, Shunstrom J. New oral anticoagulants in addition to single or dual antiplatelet therapy after an acute coronary syndrome: a systematic review and metaanalysis. Eur. Heart J. 2013; 34,1670-1680.

29. Koenig-Oberhuber V, Filipovic M. New antiplatelet drugs and new oral anticoagulants. Brit. J. Anaest. 2016; 117, ii74-ii84. 